

\section{REVISTA \\ TEORÍA Y PRÁCTICA \\ DE LA \\ ARQUEOLOGÍA HISTÓRICA LATINOAMERICANA}

ISSN: 2250-866X (impreso) | ISSN: 2591-2801 (en línea)

AÑo VI, Volumen 6, Verano DE 2017

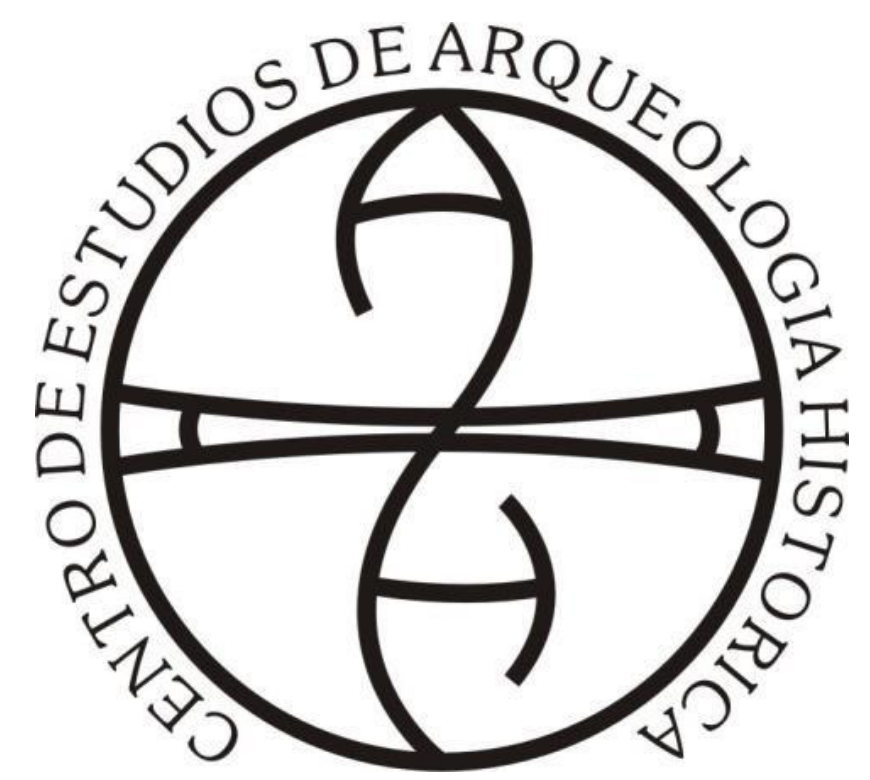

Centro de Estudios de Arqueología Histórica

FACULTAD DE HUMANIDADES Y ARTES | UNIVERSIDAD NACIONAL DE ROSARIO 

PARTICIPA EN LA RED DE ESTUDIOS INTEGRADOS SOBRE LOS PAISAJES SUDAMERICANOS

(Universidad Nacional de Rosario, Universidad Nacional de Río Cuarto,

Universidad Nacional de San Juan, Universidad de la República, Universidad Nacional de Trujillo)

AUTORIDADES DE LA UNIVERSIDAD NACIONAL DE ROSARIO

Rector: Dr. Arq. Héctor Floriani

Vicerrector: Lic. Fabián Bicciré

Secretario general: Lic. Mariano Balla

Secretario académico: Dr. Carlos A. Hernández

\author{
AUTORIDADES DE LA FACULTAD DE HUMANIDADES Y ARTES \\ Decano: Prof. José Goity \\ Vicedecano: Prof. Marta Varela \\ Secretaria Académica: Dra. Liliana Pérez
}

AUTORIDADES DEL CENTRO DE ARQUEOLOGÍA HISTÓRICA

Directora: Dra. Ana María Rocchietti

Secretaria: Prof. Nélida De Grandis

Prosecretaria: Lic. Marianela Biscaldi

Revista del Centro de Estudios de Arqueología Histórica

Teoría y Práctica de la Arqueología Histórica Latinoamericana Actas del Sexto Simposio de Arqueología Histórica Latinoamericana

Presidente del Simposio: Licenciada Mónica Valentini
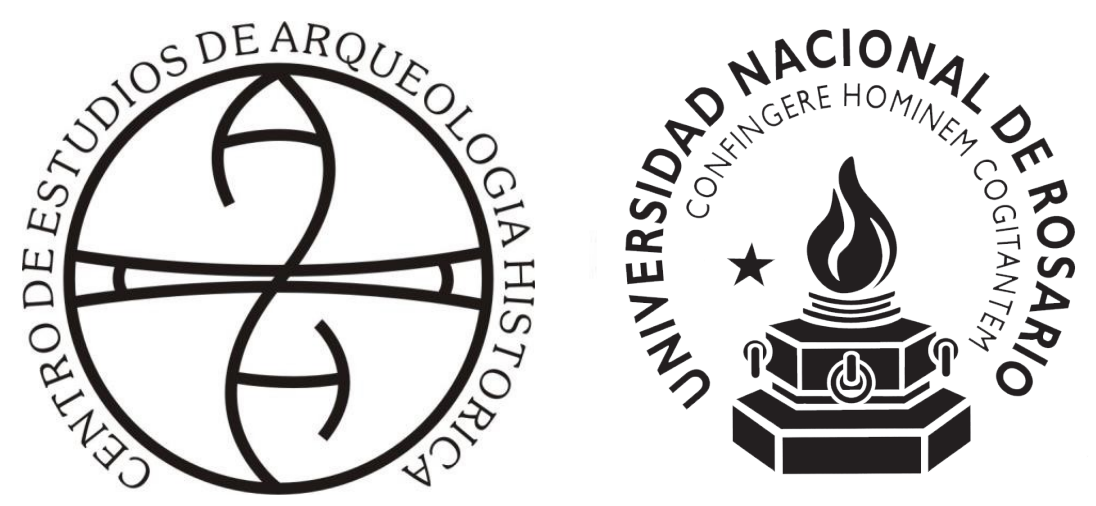


\section{Directoras Editoras}

Ana María Rocchietti y Nélida De Grandis

\section{Secretaria}

Irene Dosztal

\section{Comité Editor}

Ana María Rocchietti, Mónica P. Valentini

Nélida De Grandis, Marianela Biscaldi

Irene Dosztal, María Victoria Roca,

Fátima Solomita Banfi

\section{Comité Científico del V Simposio}

Dra. Nidia Areces

Dr. Raúl Bolmaro

Dr. Luis María Calvo

Lic. Juan Castañeda Murga

Lic. Carlos Ceruti

Dr. Juan Bautista Leoni

Dra. Eugenia Néspolo

Lic. Ruth Adela Poujade

Ing. Adrián Pifferetti

Dr. Mariano Ramos

Dr. Daniel Schávelzon

Dra. Marcela Tamagnini

Dra. Alicia Tapia

\section{Comité Científico}

Dr. Raúl Bolmaro (Universidad Nacional de Rosario)

Dr. Luis María Calvo (Museo Etnográfico de Santa Fe y Parque Arqueológico de Santa Fe La Vieja)

Lic. Carlos Ceruti (CONICET)

Dra. Dora de Grinberg (Facultad de Ingeniería, UNAM)

Dra. Eugenia Néspolo (Universidad Nacional de Luján)

Ing. Tulio Palacios (Comisión Nacional de Energía Atómica)

Lic. Ruth Poujade (Programa Misiones

Jesuíticas - Provincia de Misiones)

Dr. Mariano Ramos (Universidad Nacional de Luján)

Dra. Ana María Rocchietti (Universidad

Nacional de Rosario)
Dr. Daniel Schávelzon (Centro de

Arqueología Urbana - Universidad de

Buenos Aires)

Dra. Marcela Tamagnini (Universidad

Nacional de Río Cuarto)

Dra. Alicia Tapia (Universidad de Buenos

Aires)

Dr. Arno Álvarez Kern (Centro Nacional de

Pesquisas - Brasil)

Dra. Noemí Walsöe de Reca (CONICET)

Lic. Mónica Valentini (Universidad

Nacional de Rosario)

\section{Evaluaron este Volumen}

Horacio Chiavazza, Mónica Valentini,

Nélida De Grandis, Mariano Ramos, Juan

Bautista Leoni, Ana María Rocchietti, Javier García Cano, Flavio Ribero

\section{Diseño y diagramación}

Eugenia Reboiro

(eugenia.reboiro@gmail.com)

\section{Curaduría}

Irene Dosztal y Ana María Rocchietti

Foto de tapa: botellas de vidrio del texto de Orsini y Padula.

\section{Propietario responsable:}

Universidad Nacional de Rosario

Facultad de Humanidades y Artes,

Centro de Estudios de Arqueología

Histórica.

Entre Ríos 758. Rosario, Provincia de Santa

Fe (2000). Argentina.

Telf.: +54 (0341) 4802670

E-mail: ceahunr@gmail.com

Decreto Ley 6422/57 de Publicaciones Periódicas 


\section{Índice}

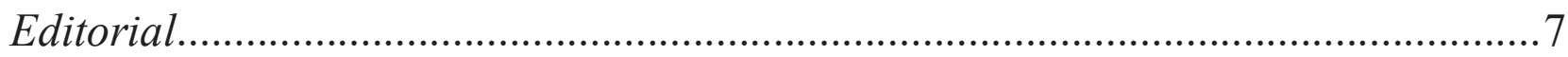

Experimentación, transferencia tecnológica y conservadurismo: notas sobre la introducción del aforro de cobre en los barcos de madera del siglo XVIII......

Nicolás C. Ciarlo

Arqueología social latinoamericana y los desafios de la arqueología urbana.

Ana María Rocchietti

"No todo fue hispánico en la casa de los Larreta". Intervención arqueológica en el jardín del Museo Larreta.

Ricardo Orsini y Horacio Padula

La construcción con ladrillo asentado en barro. El caso de la ciudad de

Rosario

Adrián A. Pifferetti

“Mientras las gárgolas nos miran”. Un sitio arqueológico urbano ¿y religioso?

en la ciudad de Luján. Provincia de Buenos Aires.

Mariano Ramos, Alejandra Raies y Matías Warr

Asentamientos europeos en la cuenca del Río de la Plata: primeras

aproximaciones a Corpus Christi.

Fernando Balducci, Julieta Sartori, Paula Galligani, Fernando Roggero, Macarena Riberi y Abril Castagnola

"De lugares y objetos". La visibilización del pasado afro en el norte de Argentina. Caso de estudio: la ciudad colonial de San Miguel de Tucumán.... 79

Luciana Chávez

Teledetección espacial aplicada a la búsqueda de restos de la Estancia San Miguel del Carcarañá.

Fabián Bognanni, Mónica P. Valentini, Mariano Darigo, Matías Warr y María Lara Moschetoni 
Rescate arqueológico. Hotel Pensión Europea. Posadas.

Misiones. Argentina.

Marianela Biscaldi y Lorena Salvatelli

El Fuerte Independencia revive tres siglos después en medio de un debate sobre sus túneles

Julio Fabián Merlo y María del Carmen Langiano

Estudio de muestras metálicas de vado de Las tres cascadas, Río Cuarto, Córdoba

Adrián A. Pifferetti

Clasificación de botones Prosser y su potencial como indicador

cronológico. Arqueología urbana de Rosario (sitio La Basurita)

María Fernanda Bruzzoni y Sandra Escudero

Una casa del siglo XX y un fuerte del siglo XVI. Arqueología

histórica en Cobos.

Jorge Esteban Cabral Ortiz y Rosana Jimena Flores

Un fechado intrigante

Mario Silveira y Mónica Patricia Valentini

Criterios adoptados para la conservación de los restos de estructura naval del Pecio Zencity de Puerto Madero (Buenos Aires, Argentina): ¿¿RE-ENTERRAR PARA CONSERVAR?!.

Eva Tavella 


\title{
ARQUEOLOGÍA SOCIAL LATINOAMERICANA Y LOS DESAFÍOS DE LA ARQUEOLOGÍA URBANA
}

\author{
Ana María Rocchietti*
}

\section{Resumen}

En tiempos de la Arqueología Social Latinoamericana se dejaron sentados algunos principios para realizar esa disciplina través de su intervención en campo así como a través de su interacción con las vecindades populares urbanas. Este trabajo toma algunas ideas de una de sus representantes conspicuas: Iraida Vargas Arenas.

Palabras clave: arqueología urbana, arqueología social latinoamericana, potencial arqueológico y barriadas populares

\begin{abstract}
In times of the Social Latin-American Archaeology, some criteria were adopted to realize the archaeology across it intervention in field as well as it interaction with poplar urban vicinities. This work takes ideas of one of most eminent representatives: Iraida Vargas Arenas.
\end{abstract}

Keywords: urban archaeology, social Latin-American archaeology, archaeological potential and popular neighborhoods

\section{Introducción}

En este sentido, mi aporte quiere completar la perspectiva de esta investigadora venezolana que estima que hay una ciencia arqueológica de la sociedad.

Presento un análisis que compromete el desarrollo teórico y práctico de la Arqueología Urbana referido a la que fuera denominada Arqueología Social Latinoamericana. Exploro sus postulados y núcleo epistemológico con la intención de encontrar inspiración en un esfuerzo particular: el de Iraida Vargas Arenas. Lo hago a partir de su argumentación sobre el valor del pasado. Ella lo define como un significado, una relación y una unificación de los hechos destacando que él sirve a la identificación social y al desarrollo del poder de los actores como programa de acción política.

\section{Axiomas principios y criterios}

La Arqueología Social Latinoamericana nació de una manera coyuntural y contingente: un manuscrito de la autoría de Luis Guillermo Lumbreras cuyo título fue Arqueología como ciencia social (1974). Tardó en publicarse pero por su testimonio se sabe que circuló entre arqueólogos latinoamericanos, al principio, de una manera informal. Lo cierto es que abrió las puertas a una arqueología

\footnotetext{
* Centro de Estudios en Arqueología Histórica. Facultad de Humanidades y Artes, Universidad Nacional de Rosario. anaau2002@yahoo.com.ar
} 
de carácter sociológico aunque -más exactamente- a una arqueología marxista. Lo curioso es que articuló su bagaje teórico en torno al concepto de cultura, al que presumo Marx se hubiera negado o al menos le hubiera otorgado el lugar dudoso de la ideología: es decir, el sistema de ideas de una sociedad sobre sus prácticas y sobre el mundo en general. Al respecto, decía Luis Felipe Bate Petersen, el teórico más destacado de esta línea epistemológica:

La forma cultural es la expresión concreta del ser y la conciencia social en cada grupo humano $\mathrm{y}$, en general, en cada sociedad. La singularidad formal de la cultura se manifiesta en todo nivel: en el comportamiento de los hombres [entiéndase también mujeres] y en la objetivación material producto de la acción, así como en el reflejo y valoración en la conciencia social de su actividad o en los organismos que la regulan. (Bate 1977, p.10)

La arqueología se preocupa del conocimiento del fenómeno social en el desarrollo histórico en su totalidad (Bate 1977, p.11), (...) totalidad del proceso, que sea congruente con sus objetivos y es necesario esclarecer su ubicación y nexos en el contexto de esa totalidad (Bate 1993, p.89).

La cultura, de acuerdo con esta argumentación, pareciera constituirse en un fenómeno de conciencia o, al menos, su sede está en la conciencia. De esta manera, desde mi punto de vista, se torsiona el sentido inicial del concepto de ideología en el marxismo: un enmascaramiento del cual no es productor el individuo concreto sino la misma sociedad a lo largo de un devenir histórico en el cual luchan las clases.

Cabe señalar que la ASL tuvo lugar en tiempos de agudos conflictos latinoamericanos, de golpes de Estado y de ascenso enfervorizado del marxismo y del populismo. Por entonces los principales temas de las Ciencias Sociales eran urbanización y subdesarrollo, ideología, razón y dominación, colonialismo y anti-imperialismo, feminismo, Estado y nacionalismo, Socialismo, campesinos y el mundo de la vida popular.

En cambio, Iraida Vargas - una venezolana dedicada a una arqueología de investigación bajo conceptos y preceptos marxistas- no se sumó completamente a esta definición del campo disciplinar ni de la misma práctica arqueológica. Ella afirmó que son las relaciones sociales el centro de la ciencia arqueológica de la sociedad y su desarrollo y transformaciones, en tanto objeto de conocimiento (Vargas 2005). Esto implica -en contrario- desplazar el foco de estudio a las relaciones sociales y las implicaciones estructurales que ellas significan. Se trataría de una ciencia sobre la sociedad, no sobre la cultura. Esto hace de Iraida Vargas un miembro hasta cierto punto discrepante del conjunto académico y político que existió y, quizá, todavía existe, bajo el rótulo ASL, aun cuando ella no lo reconociera explícitamente; de ahí mi interés en ella y sus conceptos.

\section{Iraida Vargas}

Iraida, y su compañero Mario Sanoja, en Venezuela tenían un contexto científico caracterizado por el cultivo de la ambición explicativa del marxismo como lucha por la descolonización de las periferias mundiales; por el auge de perspectivas contrarias, fragmentadas y diversificadas con influencia neoliberal y de la filosofía posmodernista europea y norteamericana y por la lenta construcción de recuperación de la utopía expresada por el ideal de una República bolivariana ${ }^{2}$.

En donde Iraida manifestó más claramente su perspectiva es un trabajo del año 1987 y no dedicado precisamente ni a la arqueología histórica ni urbana sino a las que llama sociedades preclasistas (es decir, de cazadores y recolectores). En él hace una reseña de las posiciones teóricas, por entonces en alza, de la Nueva Arqueología (Binford como principal exponente), Arqueología Sistémica (Flannery) y Arqueología biologicista (Meggers), las cuales hacen uso del término cultura con el cual ella discrepa. Para la primera la "cultura" es un fenómeno extra-somático para la adaptación; en el caso de la segunda la cultura es un subsistema respecto al Sistema total y en el del tercero se destaca el 
determinismo ambiental. Todas aplican a la Arqueología las metodologías de las Ciencias Naturales y explican el cambio social por factores externos y contingentes, dice Iraida.

A continuación, contrapone 1. Las determinaciones y las condiciones de transformación histórica, 2. La expresión de las determinaciones y las condiciones del cambio social en las formaciones preclasistas (y aplicables a las clasistas) y, finalmente, 3. Los planteos en torno a las categorías y conceptos de que nos valemos para entender y explicar la relación hombres - objeto de trabajo y de hombres entre sí. Para Vargas, el hombre es un fenómeno de la Naturaleza pero el hombre tiene ser social; es decir interrelación con el ambiente y de los hombres entre sí. Es el trabajo el que reproduce las formas de vida y a los hombres mismos. Las formas de relación en torno al trabajo no operan azarosamente sino que están reguladas por las leyes sociales, las cuales son determinantes en la acción humana aun cuando el hombre también está sometido a las leyes biológicas generales y particulares. La sociedad es siempre un fenómeno en desarrollo, un proceso infinito e inagotable.

Ahora bien, las relaciones sociales siempre son contradictorias (no importa cuán compleja pudieran resultar), es un escenario de tensiones, de manipulaciones, de oposición de contrarios. A medida que la sociedad se complejiza disminuye el acondicionamiento (la adaptación). La Naturaleza es siempre el objeto de trabajo del hombre. Los opuestos son hombre - Naturaleza, relaciones de producción y propiedad. Cuando aparece una contradicción principal nueva -como en el caso de domesticación y formación de rebaños, cambia la formación social. El trabajo objetivado en la tierra y en los rebaños (capital comunal agrario) incide para que comience la desigualdad social y las clases sociales.

Esta postura -que se advierte en la terminología de sus trabajos- se abre a un análisis fundamentado en las formaciones sociales para todo tipo de sociedad, incluidas las de cazadores. Para comprender y explicar ese proceso, Iraida Vargas propone las categorías de Modo de vida (praxis particular de la Formación Social) y Modo de Trabajo (praxis particular del Modo de Vida integrado por una combinación de ambiente, base productiva e interacción social y bajo distintos Modos de Trabajo que pueden coexistir (Vargas Arenas 1987).

Asimismo, esta posición teórica se plantea, de manera explícita, una postura ética que implica asumir un compromiso político con la sociedad en estudio. Tal compromiso supone, entre otras cosas, practicar una arqueología que trascienda un campo de acción centrado en el pasado y que busque analizar las causas que originan las actuales condiciones de existencia de las sociedades de América Latina, las formas de estructuración y desarrollo de dichas condiciones y los procesos de particularización que llevan a cada país a ser lo que es (Vargas 2005).

Dentro de su propuesta destaca el estudio de la vida cotidiana, que permite ofrecer explicaciones sobre las actividades diarias, las relaciones interpersonales, el comportamiento consuetudinario en las distintas épocas; así mismo permite conocer la ruptura de lo consuetudinario, la creación y alteración, la transformación de cada época histórica. Dicha transformación social requiere, de manera necesaria, la existencia en el colectivo de una conciencia histórica, de un conocimiento de sus procesos históricos, de la aceptación del pasado como propio. De alguna manera, se trata de romper la homogeneización de la conciencia surgida como paradigma de la expansión capitalista.

Caracas, el mundo popular y las mujeres

Iraida escribió:

Como resultado de nuestras investigaciones en el Proyecto de Arqueología Urbana de Caracas $[. .$.$] hemos podido inferir que en la organización física del espacio doméstico en Venezuela,$ y posiblemente en el Caribe oriental, existe una lógica lineal: aquellos espacios más cercanos a la puerta del frente están reservados para exhibir y ejecutar la actividad social del componente dominante del grupo doméstico. Aquellos más alejados, están reservados para los lugares donde se realizan las tareas domésticas, como son el procesamiento y cocción de alimentos, lavado de la ropa, y también para servir como área dormitorio de los sirvientes domésticos y área de disposición de la basura producida por el grupo doméstico como un todo. (Vargas 2005, p.76) 
En los sitios coloniales, puesto que la vida doméstica tiende a ser autárquica, el depósito arqueológico muestra la utilización en la vida cotidiana de muy pocos bienes importados o exóticos, principalmente mayólica y licores y muy raramente textiles, botones, objetos de metal, objetos rituales, etc. La multiplicidad de tareas necesarias para realizar los rituales comunes de cocinar, servir y consumir comidas, por ejemplo, revela la utilización de manufacturas locales criollas.

En los sitios republicanos, el consumo diversificado de bienes manufacturados revela la creciente dependencia del grupo doméstico de bienes manufacturados extranjeros y en ocasiones exóticos, esto permite la identificación en el registro arqueológico de funciones más específicas del componente doméstico dominante, como la utilización de juguetes elaborados industrialmente, objetos para la salud personal y el uso de productos farmacéuticos, el empleo de maquinarias, instrumentos de iluminación, etc., lo que ayuda a identificar el impacto, diferencias que la primera y segunda revoluciones industriales tuvieron en la naturaleza de la vida diaria de los grupos domésticos y su ruptura con la cultura criolla tradicional, lo que condujo hacia un modo de vida más cosmopolita.

De ahí que cada proyecto político haya construido nuevas significaciones y nuevas concepciones para concebir ese pasado como forma de ejercer el control sobre los individuos; en consecuencia, ha reinventado nuevas formas de tradición cultural, ha creado diferentes nociones de pueblo, ha establecido la idea de rupturas en procesos continuos, ha borrado personajes y acciones colectivas, ha seleccionado otros, ha creado nuevos elementos culturales, en suma ha elaborado en cada momento los elementos para gestar un nuevo contenido en la memoria histórica.

La calidad de vida es multidimensional pero en la ciudad se combinan el poder popular e identidad cultural de modo tal que, "en la conformación de las subjetividades participan todas las características del sujeto, las particularidades de su herencia biológica y social, el proceso de su socialización, su origen, motivaciones, gustos, inclinaciones y otras muchas" (Vargas 2007, p.04).

Por esa razón, la Arqueología urbana (o quizá sería mejor denominarla Arqueología en el seno de la actual Caracas), el estudio de la vida cotidiana, permite ofrecer explicaciones sobre las actividades diarias, las relaciones interpersonales, el comportamiento consuetudinario en las distintas épocas; asimismo permite conocer la ruptura de lo consuetudinario, la creación y alteración, la transformación de cada época histórica.

Se configura así una Arqueología del capitalismo o, también, Arqueología de la desigualdad estableciendo una antinomia u oposición de contrarios: Arqueología de sitios policlasistas vs Arqueología de los sectores corporativos de una estructura social oligárquica.

Una síntesis del pensamiento de Iraida Vargas contiene: 1. Arqueología como ciencia de la sociedad, 2. Arqueología de los contextos socio-históricos del pasado y 3. Compromiso político con la sociedad en estudio. Para lo cual aporta como metodología los siguientes pasos:

- Captar la estructura y dinámica de los sitios

- Medir el tiempo y el desarrollo social para cada momento histórico

- Encontrar las relaciones asociativas en los contextos

- $\quad$ Estudio de la producción del espacio en la sociedad capitalista

Pero es necesario comentar esta tesis y su práctica. La Arqueología latinoamericana tiene bajo su mira a los tiempos prehistóricos, precolombinos, coloniales, republicanos y contemporáneos. La Arqueología Urbana está estrechamente ligada a la Arqueología histórica pero de manera diferenciada a su práctica en los países anglosajones (Arqueología del capitalismo) y europeos (Arqueología de la historia europea no capitalista y capitalista). Las formaciones sociales en América Latina tienen un pasado menos definido porque combinan elementos étnicos muy distintos pero finalmente entrelazados o mezclados y otro tanto puede decirse de su base económica y de su cultura material que combina bienes indígenas, medievales europeos e industriales (Domínguez y Funari 2002).

En la misma dirección se han desarrollado los estudios de la interacción entre indígenas, europeos y africanos, en la creación de nuevas sociedades coloniales o postcoloniales. Conceptos de la teo- 
ría social latinoamericana fueron, en este sentido, vitales, en particular términos como transculturación, hibridización o creolización. Tales conceptos, aunque surgidos en Latinoamérica, reflejan asimismo las discusiones del postmodernismo sobre el carácter fluido, mutante o nómada de las identidades sociales. (Funari 2008, p.14)

\section{Arqueología de la desigualdad: basureros policlasistas}

Toda ciudad tiene sus basureros, cada vez más extensos e indeseables de acuerdo con el punto de vista tendencial de una sociedad que consume cada vez más, que produce cada vez más desechos y que los arroja rápidamente de los hogares sin preocuparse por su destino final. Todo basurero contemporáneo es una mezcla policlasista de aquello que en la ciudad se encuentra más o menos separado por barreras invisibles. Se trata de una coexistencia contingente, precaria y, asimismo, transitoria dado que los gobiernos municipales o regionales aplican políticas de descarte, reciclado y enterramiento. Así ocurre en Rosario: ciudad sin fundación, poscolonial, agregado de vecinos y oratorio católico, receptora de un alud de inmigrantes y foco de rebeliones y luchas de signo obrero. Ella también es una mezcla cosmopolita (Cf. Álvarez 1981).

Los basurales, como cualquier otra instalación humana, son el producto de impactos secundarios, impactos de corto y largo plazo y de impactos acumulativos. El más curioso de los basurales de Rosario fue La Lagunita.

La Lagunita ${ }^{3}$ era un cuerpo de agua ubicado a la altura de la intersección de las calles Ayolas y Teniente Coronel Sánchez, en el predio del Puerto de Rosario y en la jurisdicción del Distrito Sur, por dentro de su alambrada perimetral. Su formación se debía a un área de ingreso de agua del colindante río Paraná, encerrada por las construcciones (muelle y edificios portuarios). En ese lugar se formó un verdadero micro-ecosistema con vegetación y fauna propias del litoral fluvial, discordante con el actual paisaje de cemento, en una zona cuya impronta principal es la autopista que facilita el acceso sur a la ciudad. En torno a La Lagunita se desarrolló (bajo escombros y un estrato de tierra, cemento y residuos compactados) un antiguo basural, conocido en el barrio como Basural de Jesús Pérez y consagrado por la novela de Rosa Wernicke como Las colinas del hambre. La misma recoge la historia popular de Pérez, un ciruja devenido en magnate de la basura, quien construyó su mansión en la barranca del río, junto mismo a los depósitos que fueran su fortuna. El basural, entonces, constituía un sitio arqueológico a cielo abierto, en la costa, del tipo habitual para el trabajo arqueológico en contexto urbano pero sostenía también una historia -en parte leyenda- de los pobres urbanos que vivían excavando en las basuras para obtener su jornal (Figura 1).

El caso admite algunas reflexiones de índole conceptual en relación con el trabajo arqueológico en y de las ciudades. Unas tienen vinculación con los límites del fenómeno urbano y con los límites de los sitios arqueológicos en sí mismos; otras al registro arqueológico como tal y por fin, algunas a la responsabilidad de la intervención en ellos. Por esa razón el análisis de Iraida Vargas y las metas de la ASL son pertinentes para encontrar otras dimensiones a un trabajo relativamente rutinario ya que los basurales casi no admiten exámenes estratigráficos (la secuencia vertical es caótica, no ofrece discontinuidades y todo el contenido se transforma en un ambiente anaeróbico). Las otras dimensiones son los trabajadores en el basural y la barriada popular configurando un modo de vida, interrumpido y cambiado por la extensión de los loteos, la obra pública, la construcción de una estación de descarga $\mathrm{y}$, por fin, el trazado de una vía de tránsito rápido que hace anónimo el sector portuario.

Urbanistas y cientistas sociales reconocen, hoy, que el fenómeno urbano va tomando en todo Occidente características especiales, derivadas de las nuevas tecnologías de la comunicación y del régimen de ocupación del suelo por las empresas productivas. Toda ciudad desenvuelve sus funciones espaciales de acuerdo con un estilo de organización de cultura en el territorio y, por otra parte, ellas evolucionan cambiando las características de los bienes, los servicios y de la población. Las ciuda- 


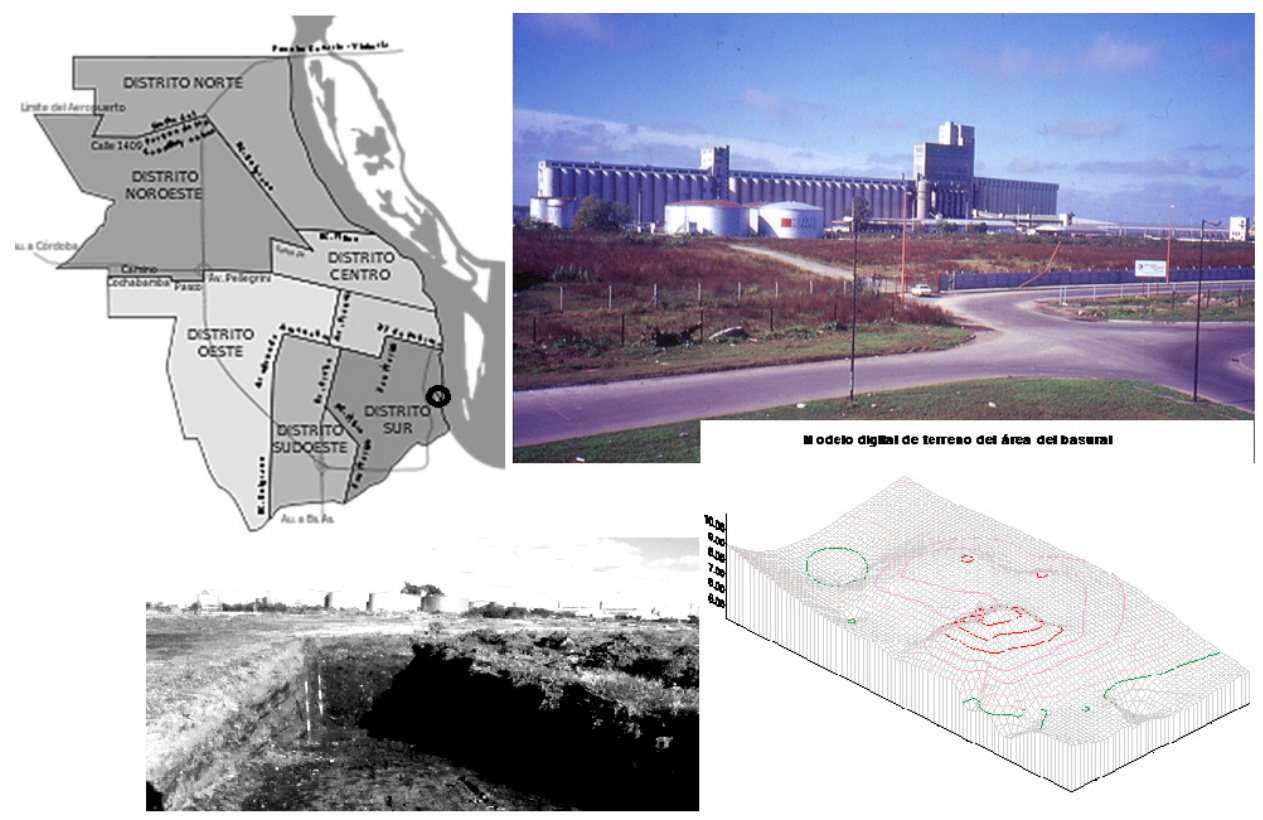

Figura 1: La Lagunita. Puerto Rosario

des son el resultado de las formaciones económico-sociales características del siglo XX que pueden caracterizarse como industriales y post-industriales. Rosario participa todavía de ambas cualidades y exhibe tendencia al crecimiento territorial por aporte de nuevas migraciones llegadas especialmente del litoral pobre del norte santafesino, de Chaco, Formosa y Corrientes.

Asimismo, una fracción de la ciudad está actualmente sujeta a cambios de diseño e infraestructura por presión de la inversión privada y municipal que acabará en el remodelamiento radical de la costa y de una parte de sus barriadas. Estos impulsos son cíclicos en la política urbana y pueden detectarse sus sucesivas etapas como superposición de marcas en su paisaje arquitectónico. Hay que señalar que, en este sentido, Rosario casi siempre se ha volcado hacia el río ya sea por grandes obras de infraestructura o por la construcción de lugares de esparcimiento o adorno. El Distrito Sur es una jerarquía territorial (Municipalidad de Rosario 2000) con una realidad social heterogénea sometida en estos días a una intensa remodelación capitalista que impactará sobre la población y sobre el paisaje.

Las ciudades, hoy, comienzan a ser en América Latina -fruto de la economía y de las tendencias culturales- espacios difusos, cada vez más indeterminados (Haas 1999) en los que además del espacio físico existe un espacio inmaterial, no localizado, des-territorializado -por así decirlo- por la instantaneidad de las comunicaciones, la descentralización de los servicios hacia la periferia y la concentración de las decisiones en mercados segmentados (Castells 1995).

Si esta tendencia culmina ya no habrá muros y extra-muros, centro y periferia como en el tiempo de Jesús Pérez y el basural. Este tipo de ciudad acentúa las desigualdades y la marginación, da lugar a una producción cultural heterogénea y aporta un complejo desarrollo territorial. El centro de la ciudad podría estar en cualquier parte.

Estos fenómenos sociales tienen una consecuencia importante en la práctica de la arqueología ya que la tradicional división entre arqueología de Centro Histórico y la arqueología de "extramuros" (arqueología de fundadores o de elite en relación con arqueología de periferia o de sectores populares) daría lugar a una arqueología distrital-administrativa menos vinculada a las clases sociales y a su espacialización, a las geografías diferenciadas del capital y el trabajo. 
En definitiva, ¿qué es el potencial arqueológico de una etapa en la historia y en la sociología de una ciudad?

El potencial arqueológico urbano se demarca por el acceso a los sitios arqueológicos (a través de su visibilidad tanto como por las referencias documentales), por el impacto que la vida de la ciudad ejerce sobre ellos y por el posible régimen de museificación es decir, lo posible o imposible de escenificar para interpretar el pasado agregando a la ciudad lugares "didácticos" en los que la hegemonía de la clase dominante se hace patente mediante mensajes de identificación. El potencial depende del crecimiento urbano (que borra las huellas de la historia) y por las nuevas tecnologías (de movilidad y de producción) que re-localizan los lugares significativos para la población.

El potencial arqueológico de la ciudad sirve indudablemente al pasado pero mucho más al futuro si consideramos la problemática del patrimonio cultural, la deontología de la profesión de los arqueólogos, las alternativas constructivas urbanas y la nueva territorialización.

La escala territorial de los sitios arqueológicos en contexto urbano está vinculada con la escala territorial de la ciudad: sus dimensiones y su visibilidad varían en proporción directa con el poder expansivo y destructivo de las actividades urbanas y, en esta dirección, cuenta considerar la alternancia entre espacios construidos y espacios libres.

Los primeros devienen del uso del suelo y del régimen de propiedad tal como se ve ahora en Parque Italia, donde el proyecto de parquización expulsa vecinos y transforma en arqueología lo que fuera un asentamiento popular. Los espacios libres desarrollan los verdaderos extra-muros (aún cuando no lo sean por emplazamiento o por función) ya que la pobreza urbana hace de los basurales un paisaje prácticamente rural con el apacentamiento de caballos y con viviendas y sus entornos de uso extraídos del patrón rural de vida.

Es decir que se asiste a la conjugación de materialidades ofrecidas por la ciudad y por los sitios arqueológicos y a la contingencia de que se tornen efímeros (Schávelzon 1999).

El registro arqueológico resulta así en una estructuración continua cuya situación contextual varía en aislamiento, interdigitación, contigüidad o discontinuidad. La Lagunita se entramaba con la instalación portuaria, con las viviendas no erradicadas que colindaban con una villa miseria (Koldorf et al. 2001 a y b; Simonassi 2000; Rocchietti et al. 2004).

El estudio del potencial arqueológico consiste, asimismo, en una evaluación de impacto ambiental que tiene el valor de ser un instrumento para la preparación de decisiones, haciendo previsibles las consecuencias de un sistema ambiental compuesto por el medio social y natural así como por las interacciones entre ambos (CEPAL, 1991; Pirez, 1991) Incluye la definición de las áreas de estudio, la identificación de los efectos, la predicción de alteraciones ambientales, la propuesta de ordenamiento preventivo, la evaluación de impactos y la propuesta de ordenamiento ambiental.

Por tanto, en la dialéctica entre sitio-objeto y sitio imaginado se puede advertir que no existen sitios aislados, todos forman una cadena de espacio urbano, que existen en su materialidad y en la narración, que no existen sitios - memoriales para la historia popular y que la desaparición ejerce un nuevo régimen de historicidad: la potencialidad efímera de los objetos imaginados.

Esto genera un ordenamiento por valores de sentimiento colectivo (apego, aprecio, tradiciones asociadas, etc.) como por valores derivados de la apreciación científica o histórica de los registros arqueológicos. Y con ello la estimación de la posibilidad de desarrollo de los sitios como organizadores territoriales futuros (por ejemplo, en caso de ser museificados su transformación en lugares de movimiento de gente, de inversión pública y privada, en nuevos circuitos de socialidad urbana, etc.), del valor patrimonial en términos de uso del suelo y de régimen de propiedad para cada uno, su futura evolución en la tierra urbana y de los subsecuentes factores de destrucción, alteración o modificación y, por último, la factibilidad del trabajo arqueológico en sí mismo. Todas estas dimensiones del potencial lo ligan fuertemente con problemáticas sociales haciendo un claro nexo entre la arqueología y la cuestión social. 
De todos modos, permanece la incógnita sobre si la simple vecindad o coexistencia de la barriada popular deviene en identidad y empoderamiento de los actores y de su espectro de asociaciones informales. Probablemente se requiera algo que Iraida no contempla o no aborda: la acción de agentes partidarios o estatales que los afilien a un proyecto de tal empoderamiento o, al menos, participación. No siempre la empresa científica se embarca en ese itinerario; requiere mucho más que un proceso educativo o de inclusión de vecinos y esto es una finalidad explícitamente política y no neutral.

Perdura la definición de Lumbreras (1990) sobre la razón colonial: una relación desigual entre dos pueblos: uno dominante y otro dominado, cada uno con su racionalidad y patrimonio propio; el patrimonio es su existencia histórica, que la ha hecho posible y que garantiza su existencia. La matriz de esa racionalidad la coloca en la conciencia colectiva, en la imagen de sí mismo. Iraida Vargas, en cambio, coloca el problema en la lucha de clases.

\section{Consideraciones finales}

Iraida Vargas sostiene que la vida cotidiana es el centro del acaecer social, que la vida material se concreta en la vida cotidiana y que el espacio social es producido y reproducido en las relaciones sociales. La arqueología de los basurales se asoma a un ser social obrero, histórico y contradictorio que ha existido y existe bajo la premisa del trabajo objetivado pero no en la propiedad sino en el acarreo y en la exploración de la basura mientras el cambio social lo deja obsoleto.

La ASL dejó enseñanzas sobre la singularidad de lo popular, sobre el hecho de que la arqueología converge en el conocimiento "realista" de las bases sociales populares y con la captación -por la arqueología- de la acumulación de energía social en el mundo del trabajo y su ideología de clase.

\section{Notas}

${ }^{1}$ En los países en los que más éxito tuvo la ASL fueron Perú, Chile y Venezuela en los que los gobiernos de Juan Velasco Alvarado, de Salvador Allende y de Carlos Andrés Pérez marcaron el período y, de alguna manera, el destino político y económico regional de las décadas posteriores.

${ }^{2}$ Cuya historia iba a culminar con el gobierno de Hugo Chávez y las peripecias que se vivieron después en ese país.

${ }^{3}$ Pude llevar a cabo el Programa Potencial Arqueológico y Socio-cultural de la Ciudad de Rosario (Secretaría de Ciencia y Técnica de la Universidad Nacional de Rosario, Argentina) gracias a la Asociación Vecinal del Distrito Sur (Rosario de Santa Fe), a los colegas que lo integraron y a los estudiantes de Escuela de Campo (Cátedra Metodología de la Investigación III Orientación Arqueología) Departamento de Arqueología, Escuela de Antropología, Facultad de Humanidades y Artes. Universidad Nacional de Rosario.

\section{Referencias}

ÁlVAREZ, J. (1981). Historia de la ciudad de Rosario. Santa Fe: Universidad Nacional de Litoral. BATE, L. F. (1977). Arqueología y Materialismo Histórico. México: Ediciones de Cultura Popular BATE, L. F. (1993). Teoría de la cultura y arqueología. Boletín de Antropología Americana. México: Instituto Panamericano de Geografía e Historia.

CASTELLS, M. (1995). La ciudad informacional. Tecnologías de la información, re-estructuración económica y el proceso urbano-regional. Madrid: Alianza. 
CEPAL (1991). Evaluaciones de impacto ambiental en América Latina y el Caribe. Santiago de Chile: Comisión Económica para América Latina.

DOMÍNGUEZ, L. y FUNARI P.P.A. (2002). La arqueología urbana en América Latina: el caso de La Habana Vieja, ciudad arqueológica. Etudos Ibero-Americanos 28 (2), 113 - 124.

FUNARI, P. P.A. (2008). Arqueología Histórica Mundial y Latinoamericana en las últimas dos décadas. Revista Arqueología Histórica Argentina y Latinoamericana, (2) 11 - 15.

HAAS, C. (1999). El fenómeno urbano. Difusión de la ciudad y dificultades para la determinación de sus límites. Anuario de la División Geografia.

KOLDORF, A. E.; DE CASTRO R.; COLESSO S. y OLASO J. (2001a). Antropología Social y Arqueología: relevamiento y análisis del "paisaje cultural" de un barrio de Rosario. En M. A. Caggiano (Ed), Milenio. 14-28

KOLDORF, A. E.; DE CASTRO R.; COLESSO S. y OLASO J. (2001b). Interdisciplina: la antropología social en un abordaje conjunto con la Arqueología en el estudio de un espacio enclavado en un barrio histórico de Rosario. "La República de la Sexta". IV Jornadas de investigadores en arqueología y etnohistoria del centro-oeste del país y III Seminario de arqueología histórica del centro-oeste del país. Río Cuarto: Universidad de Río Cuarto: 11 - 20.

LUMBRERAS L.G. (1974). La Arqueología como Ciencia Social. Lima: Ediciones Histar.

LUMBRERAS, L. G. (1990). Consideraciones preliminares para la crítica de la Razón Colonial. En Bonilla, H., Nurger, M., Lumbreras, L. G., Menéndez-Carrión. A., Ramón G. y Tandeter E. Los Andes: el camino del retorno. $55-70$.

MUNICIPALIDAD DE ROSARIO (2000). La descentralización de la ciudad de Rosario. Rosario: Programa de Descentralización y Modernización.

PIREZ, P. (1991). Municipio, necesidades sociales y política local. Buenos Aires: Grupo Editor Latinoamericano/IED-AL.

ROCCHIETTI, A. M., DE GRANDIS N. y DE LA PENNA J. (2004). Arqueología de las ciudades. Programa de Potencial Arqueológico y Sociocultural de Rosario (Argentina). En Bechis, M. (comp) Cuartas Jornadas de Investigadores en Arqueología y Etnohistoria. Segundo Seminario de Arqueología Histórica. Facultad de Ciencias Humanas. Río Cuarto: Universidad Nacional de Río Cuarto.

SCHÁVELZON, D. (1999). Plan de Preservación del Patrimonio Arqueológico para la Ciudad de Buenos Aires. Buenos Aires: Universidad de Buenos Aires.

SIMONASSI, S. (2002). Territorio y temporalidad. El proceso de conformación del mundo del trabajo en un barrio de Rosario. En Actas de las II Jornadas de Antropología de la Cuenca del Plata. Antropología sin fronteras. (CD-ROM). Rosario: Escuela de Antropología, Facultad de Humanidades y Artes, Universidad Nacional de Rosario.

VARGAS ARENAS, I. (1987). Sociedad y naturaleza en torno a las mediaciones y determinaciones para el cambio de la Formación Económica Social preclasista. En Boletín de Antropología Americana. $65-74$.

VARGAS ARENAS, I. (2005). La Arqueología Social: un paradigma alternativo al angloamericano. RHAA, (8), $73-78$. 
VARGAS ARENAS, I. (2007). Algunas ideas sobre los consejos Comunales y la calidad de vida de las mujeres populares en Venezuela. Revista Venezolana de Estudios de la Mujer. 12 (29), 33-48

Recibido: 25/04/2017

Aceptado: 19/07/2017 